\title{
The COVID-19 Pandemic and Enzyme Replacement Therapy in Lysosomal Storage Disorders
}

\author{
(1) Asburçe Olgaç11, (1) Çiğdem Seher Kasapkara22, (1) Burak Açıkel3, (1) Yılmaz Yıldız1, \\ (1) Gülhan Karakaya Molla22, (1) Mustafa Kılıç1 \\ 1University of Health Sciences Turkey, Dr. Sami Ulus Training and Research Hospital, Clinic of Pediatric Metabolism, Ankara, Turkey \\ ${ }^{2}$ Ankara Yıldırım Beyazıt University, Ankara City Hospital, Clinic of Pediatric Metabolism, Ankara, Turkey \\ ${ }^{3}$ Konya City Hospital, Clinic of Child and Adolescent Psychiatry, Konya, Turkey
}

\begin{abstract}
Aim: The coronavirus disease-2019 (COVID-19) pandemic has caused a worldwide public health emergency, especially affecting people with chronic illnesses including lysosomal storage disorders (LSDs). The unfavorable conditions due to COVID-19 have mostly affected people with chronic conditions, in terms of disease vulnerability and access to health-care. In the present study, we aimed to assess the problems the patients with LSDs on enzyme replacement therapy (ERT) have encountered during the pandemic, and their level of anxiety. Parental evaluation has also been made for pediatric patients.

Materials and Methods: A total of 19 participants were recruited. A semi-structured interview was structured to evaluate the effects of the COVID-19 pandemic on ERT. The Turkish version of "Hospital Anxiety and Depression scale" (HADS) for adult patients and parents were used to evaluate anxiety. Patients between ages 8-17 completed the child version of the Revised Child Anxiety and Depression scale (RCADS).

Results: The study was completed by 19 patients, and 13 parents. Five patients (26\%) admitted temporary disruption of treatment, of which the most common reason was the fear of getting infected. Eighty-nine percent of all participants were willing to receive treatment at home. Only one adult patient revealed to feel anxiety (16\%). While among parents evaluated with HADS, 7/13 had scores that indicated depression and anxiety, $3 / 4$ pediatric patients had RCADS scores indicative of anxiety and depression.

Conclusion: The problems LSD patients have been facing during the pandemic, should be identified along with their attitudes regarding ERT in order to maintain the sustainability of their treatment. The psychological health of these patients should also be identified and supported, to provide optimal care to patients.
\end{abstract}

Keywords: COVID-19, lysosomal storage disease, enzyme replacement therapy, home-therapy

\section{Introduction}

The coronavirus disease-2019 (COVID-19) pandemic caused by the severe acute respiratory syndrome coronavirus-2 (SARS-CoV-2) has become a global problem, affecting nearly every country since March 2020 (1). Along with increasing cases and unavailability of a specific treatment, the health systems of countries have become overloaded with the burden of the pandemic. The disease has caused increased anxiety worldwide, also in children, due to the fear of getting infected, and suffer its severe complications.

The unfavorable conditions due to COVID-19 have mostly affected people with chronic conditions, in terms of disease vulnerability and access to health-care (2). Patients who

\section{Address for Correspondence}

Asburçe Olgaç, University of Health Sciences Turkey, Dr. Sami Ulus Training and Research Hospital, Clinic of Pediatric Metabolism, Ankara, Turkey Phone: +90 5339627800 E-mail: mabolgac@yahoo.com ORCID: orcid.org/0000-0002-4989-221X Received: 07.10 .2020 Accepted: 16.11 .2020

${ }^{\circ}$ Copyright 2021 by Ege University Faculty of Medicine, Department of Pediatrics and Ege Children's Foundation The Journal of Pediatric Research, published by Galenos Publishing House. 
are dependent on health-care facilities on a routine basis, have faced several problems due to restrictions concerning treatment and follow-up visits, since most of the hospitals have been focused on the treatment of the patients severely affected with SARS-CoV-2. Also, due to the fact that these patients are obliged to attend health-care facilities, the risk to get infected by the COVID-19 pandemic has increased (3).

People with rare metabolic disorders are also under risk during the pandemic because of multi-systemic involvement including vital organs (4). Lysosomal storage diseases (LSDs), are a group of rare, inherited metabolic disorders, that are characterized by progressive accumulation of nondigested substrates within cells, due to diminished activity of lysosomal enzymes. Although they cannot be cured, some treatment options have been emerged by the advances in the field. Enzyme replacement therapy (ERT) is administered as intravenous infusions, for every 1-2 weeks, and has been successful in many LSDs. Although hematopoietic stem cell transplantationand substrate reduction therapy (SRT) are available for a limited number of LSDs, ERT is the only approved and most effective therapy for the majority for now, and requires lifetime administration due to short halflife (5).

At the present time, Gaucher, Fabry, Pompe diseases, acid lipase deficiency, as well as mucopolysaccharidoses (MPSs) type I, II, Vla, VI and VII can be treated by ERT (Table I) (6). Although many centers provide the facilities for home-based infusions for these patients, in some countries patients need to attend hospitals to receive treatment, as in Turkey.

In the present study, we aimed to evaluate the problems regarding treatment of patients with LSDs on ERT during the COVID-19 pandemic. We have also assessed the anxiety levels of patients and parents related with the pandemic, using validated depression scales.

\section{Materials and Methods}

The study was carried on between September and October 2020. Patients with LSDs that were receiving ERT in University of Health Sciences Turkey, Dr. Sami Ulus Maternity and Child Health Training and Research Hospital were invited to participate in the study. A semi-structured interview was designed on the attitudes of patients or their parents regarding the COVID-19 pandemic. Also, the difficulties that the patients experienced during hospital admission and access to medication were evaluated. Parents were asked to complete a set of questions including age, sex, duration of ERT, the presence of COVID-19 infection within the family or relatives, contact with a COVID-19 patient, adherence to the restrictions due to the pandemic, and any problems encountered during hospital admissions for ERT or access to medications (Table II).

To assess depressive symptoms of patients between ages 7-18, Revised Children's Anxiety and Depression scale (RCADS), and patients above the age 18, and for parental evaluation, the "Hospital Anxiety and Depression scale" (HADS) were used. Both of these questionnaires have been shown to be a reliable and valid tool to assess the general population (7).

The RCADS consists of 47 items developed to evaluate DSM-IV based symptoms of anxiety disorders and depression in children and adolescents, and their parents, with response options of $0=$ never, $1=$ sometimes, $2=$ often, and $3=$ always (7). The questionnaire was validated in Turkish by Gormez et al. $(8,9)$. The answers of the questionnaire are grouped into six subscales: Separation Anxiety Disorder, Anxiety Disorder (General Anxiety Disorder), Social Phobia, Panic Disorder, Major Depressive Disorder, and Obsessive Compulsive Disorder. For each subscale, scores are given, as well as a total anxiety score, which is the sum of all anxiety, and a total score for all the scales (sum of six subscales). Higher score indicates greater depressive symptoms. Raw scores are converted to age and gender adjusted T scores, using standardized tools (7).

\begin{tabular}{|l|l|}
\hline \multicolumn{2}{|l|}{ Table I. ERTs available for various LSDs } \\
\hline LSD subtype & ERT \\
\hline Gaucher disease & $\begin{array}{l}\text { Imiglucerase } \\
\text { Taliglucerase } \\
\text { Velaglucerasa alfa }\end{array}$ \\
\hline Fabry disease & $\begin{array}{l}\text { Agalsidase alpha } \\
\text { Agalsidase beta } \\
\text { Pegunigalsidase alpha }\end{array}$ \\
\hline MPS I (Hurler-Scheie syndrome) & Laronidase \\
\hline MPS II (Hunter syndrome) & $\begin{array}{l}\text { Idursulfase } \\
\text { Idursulfase beta }\end{array}$ \\
\hline MPS IVa (Morquio A syndrome) & Elosulfase alpha \\
\hline MPS VI (Maroteaux-Lamy syndrome) & Galsulfase \\
\hline MPS VII (Sly syndrome) & Vestronidase alpha \\
\hline Acid lipase deficiency & Sebelipase alpha \\
\hline Pompe disease & Alglucsidase alpha \\
\hline Alpha-mannosidosis & Velmanase alpha \\
\hline $\begin{array}{l}\text { ERT: Enzyme replacement therapy, LSD: Lysosomal storage disorders, MPS: } \\
\text { Mucopolysaccharidose }\end{array}$ \\
\hline
\end{tabular}




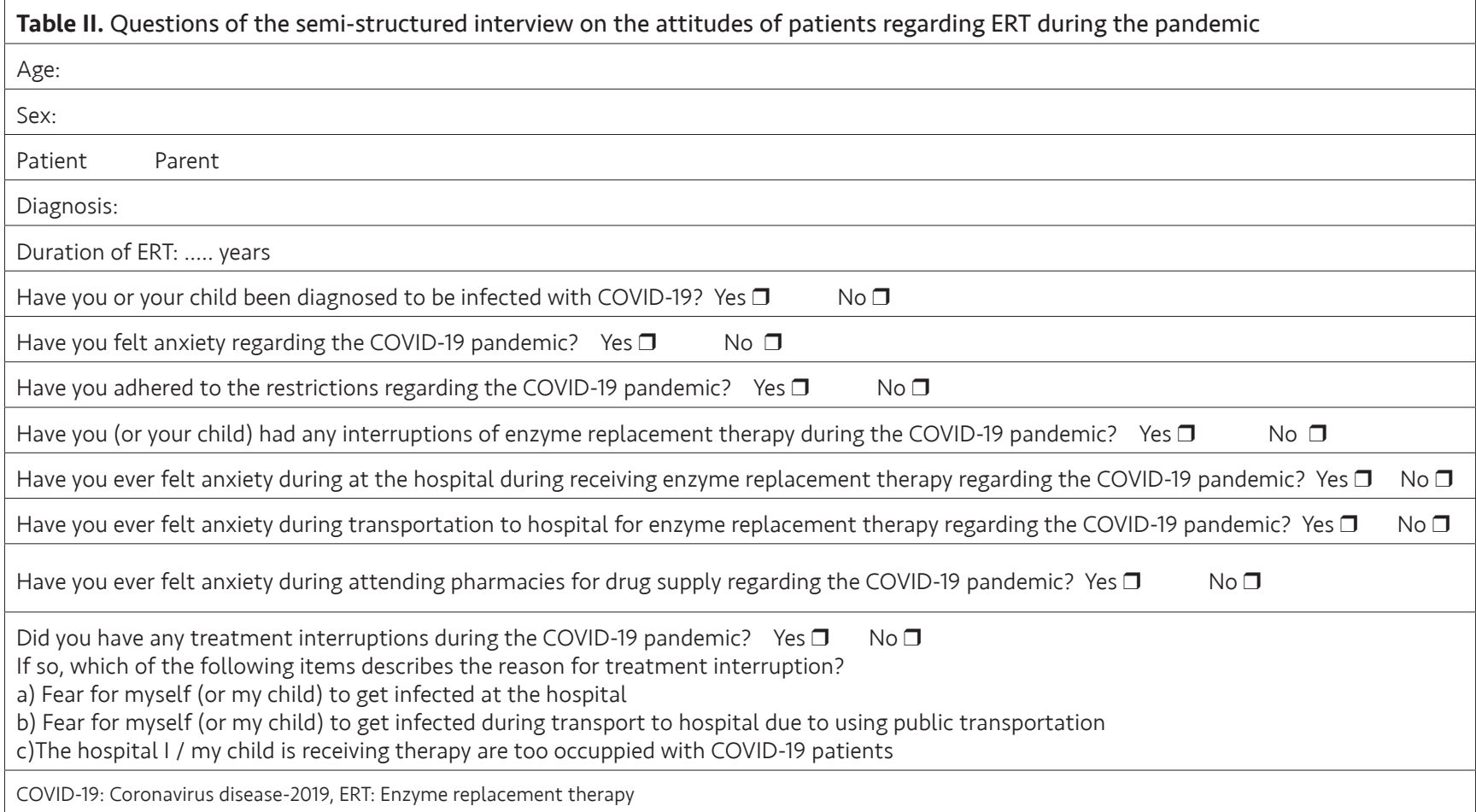

Hospital Anxiety and Depression scale (HADS) consists of 14 items divided into two subscales for anxiety and depression (7 depressive and 7 anxiety items). The items are rated on a 0-3 scale, where each items are summed to reach a total score. A score of $\geq 10$ indicates anxiety, where $\geq 7$ indicates depression (10). It is validated to be used in adolescents, and has suitable psychometric properties for this population (11).

The questionnaires were conducted in a private room by patients and parents and took about 5 minutes to complete. Patients that did not attend our clinic during the study period completed the survey via telephone. All participants provided informed consent.

Ethics approval was obtained from the ethics committee of Ankara Yıldırım Beyazıt University, on September 2020, from the local institutional review board according to guidelines of the Helsinki Declaration of Human Rights.

\section{Results}

At the time of the study, 22 LSD patients being followedup in our department, were on ERT, and thus regularly admitted to the outpatient clinic, 19 of whom accepted to participate in the study. The study was completed with 19 patients (13 pediatric and 6 adult patients) and 13 parents.

The ages of pediatric patients at ranged from 2.5 to 16 years (mean age: $7.7 \pm 4.7$ years). Male to female ratio was
(7/6). Ages of adult patients ranged from 19 to 50 years (mean age: $35.2 \pm 14.1$ ) (male to female ratio: $4 / 2$ ). Thirteen parents were also evaluated, ages ranging between 27 and 44 years (mean age 33.8 \pm 5.98 ), and male to female ratio being $4 / 9$. The demographic data of patients are given in Table III. All patients were receiving ERT (alglucosidase alfa, laronidase, idursulfase, galsulfase, imiglucerase, taliglucerase alfa, agalsidase alfa, oragalsidase beta, according to diagnosis). The patients were receiving ERT for 1 to 14 years. Mean duration of ERT was $4.5 \pm 3.2$ years (Table III).

None of the patients had a proven diagnosis of an infection with COVID-19. All patients indicated that they complied with the precautions that were being taken by the ministry of health. Three patients revealed getting into contact with a person diagnosed with COVID-19 from relatives, but had no signs of infection.

Five patients (26\%) admitted temporary disruption of treatment. Four of these patients were continuing ERT at longer intervals (once a month). The most common reason was the fear of getting infected at the hospital, or during transport to hospital due to using public transportation. One patient had missed one infusion on March 2020, due to inadequacy of hospital services dealing with the burden of COVID-19 (Table IV). Eighty-nine percent of participants were willing to receive their treatment at home during the pandemic, other than two patients, one the parent of a child 
Table III. Demographic characteristics of patients enrolled in the study

\begin{tabular}{|c|c|c|}
\hline $\begin{array}{l}\text { Demographic } \\
\text { characteristics }\end{array}$ & Patients & Parents \\
\hline \multicolumn{3}{|l|}{ Age (mean $\pm S D)$} \\
\hline Pediatric & $\begin{array}{l}2.5-16 \text { years } \\
(7.7 \pm 4.7 \text { years })\end{array}$ & \\
\hline Adult & $\begin{array}{l}19-50 \text { years } \\
(35.2 \pm 14.1)\end{array}$ & $\begin{array}{l}27-44 \text { years } \\
(33.8 \pm 5.98)\end{array}$ \\
\hline \multicolumn{3}{|l|}{ Sex } \\
\hline \multicolumn{3}{|l|}{ Pediatric } \\
\hline Male & 7 & \\
\hline Female & 6 & \\
\hline \multicolumn{3}{|l|}{ Adult } \\
\hline Male & 4 & 4 \\
\hline Female & 2 & 9 \\
\hline $\begin{array}{l}\text { Duration of ERT } \\
\text { (mean } \pm \text { SD) }\end{array}$ & $4.5 \pm 3.2$ years & \\
\hline \multicolumn{3}{|l|}{ Subtype of LSD (n) } \\
\hline Fabry disease & 6 & \\
\hline Gaucher diasease & 3 & \\
\hline MPS 1 & 2 & \\
\hline MPS 2 & 2 & \\
\hline MPS 6 & 2 & \\
\hline Pompe disease & 4 & \\
\hline
\end{tabular}

Table IV. The reasons of distruption of treatment

\begin{tabular}{|l|l|}
\hline Reason of distruption & (n) \\
\hline Fear of getting infected at the hospital & 4 \\
\hline Fear of getting infected during transport to hospital & 4 \\
\hline Hospital services loaded with the burden of COVID-19 & 1 \\
\hline COVID-19: Coronavirus disease-2019
\end{tabular}

with Pompe disease who had a history of anaphylaxis during treatment and an adult Fabry patient due to a personal feeling (fear of allergic reaction).

Nine out of thirteen parents claimed to feel anxiety during hospital admissions or regarding drug supply (69\%), among them, 7 (53\%) had HADS scores that indicated depression, and increased anxiety. Among the children of these parents, 3 had interrupted treatment during the pandemic, and the reason for interruption was the fear of getting an infection (Table V).
Table V. Anxiety and depression levels of patients and rate of treatment interruption

\begin{tabular}{|c|c|c|}
\hline & Positive* (n) & $\begin{array}{l}\text { Interruption of } \\
\text { treatment among } \\
\text { participants with } \\
\text { increased anxiety } \\
\text { and depression }(\mathrm{n})\end{array}$ \\
\hline \multicolumn{3}{|l|}{$\begin{array}{l}\text { Claim of feeling } \\
\text { anixety during hospital } \\
\text { admission for ERT }\end{array}$} \\
\hline \multicolumn{3}{|l|}{ Patients } \\
\hline Adult & $1 / 6$ & 1 \\
\hline Pediatric & $2 / 4$ & \\
\hline Parents & $9 / 13$ & \\
\hline \multicolumn{3}{|l|}{ HADS score } \\
\hline Adult patients & $1 / 6$ & 1 \\
\hline Parents & $7 / 13$ & $3^{* *}$ \\
\hline \multicolumn{3}{|l|}{ RCADS score } \\
\hline $\begin{array}{l}\text { Patients } \\
\text { (Aged 8-17) }\end{array}$ & $3 / 4$ & 2 \\
\hline
\end{tabular}

Among 6 adult patients, only one (who is also the mother of one pediatric patient) had scores indicative of anxiety and depression, who had also interrupted treatment. The same patient revealed to feel anxiety of getting infected during hospital admissions or at pharmacies (16\%).

Among patients aged 8-17 that were evaluated by RCADS (4 patients), sub-scores of 3 patients (75\%) were found to be above the threshold level and one of these 3 patients also had high total anxiety and depression scores. Two of these patients had also interrupted treatment due to fear of getting infected during receiving ERT.

\section{Statistical Analysis}

Statistical analyses regarding the relationship between interruption of treatment and anxiety scores were not performed due to insufficient number of patients enrolled in the study.

\section{Discussion}

The COVID-19 pandemic due to SARS-CoV-2 caused weaknesses in the world-healthcare systems of countries, pre dominating rare and chronic conditions including inborn errors of metabolism $(1,2)$. Particularly, patients with inborn errors of metabolism (IEMs) can be considered at high risk for severe SARS-CoV-2 infection, since they often suffer 
from a multisystem disease, including respiratory and cardiovascular system involvement (12).

It is well known that SARS-CoV-2 uses the lysosomal system to infect cells (13). Therefore, patients with LSD may be at increased risk for infection and complications of SARSCoV-2, because of lysosomal dysfunction, and activation of inflammatory cascades (14).

Our study group consists of a vulnerable population, not only due to the effects of the disease itself, but also being dependent on health-care services in terms of treatment. Neither of our patients had a proven diagnosis of COVID19. This may be due to the strict adherence of patients to the rules for prevention of infection. It is impossible to generalize our findings, since tests were not done on a routine basis, and asymptomatic cases could not be ruled out. Previous studies reported in the literature have also reported low incidence of COVID-19 among LSD patients $(12,15)$.

Scarce amount of studies exist in the literature addressing LSD patients during the COVID-19 pandemic. Mistry et al. (14) have published guidelines for the management of Gaucher disease patients and have indicated that ERT should be continued regularly, and interruptions should not be extended, even in infected patients, to avoid aggravation of symptoms due to withdrawal of treatment and also support the vital organ systems, in case of infection. The authors have stated that, interruption of therapy for weeks to months could be tolerated in stable patients. Unfortunately, it is impossible to generalize these guidelines to other subtypes of LSDs, since each LSD is unique in its own clinical findings and progression.

A study by Andrade-Campos et al. (12) revealed that, among 48 Gaucher disease patients receiving hospitalbased ERT, 11 (25\%) reported therapy interruptions related with the outbreak, while patients on oral SRT continued to receive treatment regularly. Sechi et al. (16) have reported 102 patients with various types of LSDs, of whom 71 were receiving ERT during the pandemic. While, patients receiving home-based ERT continued their treatment regularly, disruptions occurred in $49 \%$ of patients receiving ERT in the health-care centers (16).

Unfortunately, home-therapy is not currently available in Turkey. Thus, all patients recruited in our study were on hospital-based treatment. The rate of interruption of treatment was $26 \%$ in our study, similar to the study reported by Andrade-Campos et al. (12). Obviously, our study population does not reflect the overall situation in our country. Turkey has been suffering from the devastating effects of the pandemic, and the rate of interruption of treatment among LSDs may be much higher.

In a recent report, Elmonem et al. (4) have analyzed 16 centers taking care of IEMs during the COVID-19 pandemic. According to their report, $88 \%$ of centers have limited the numbers of routine patients' visits per day and $20 \%$ of have referred patients to other IEM clinics. Also, travel restrictions have affected the follow-up of patients of $93 \%$ of participating centers. The authors have specified the most important factor of treatment interruption to be the fear of the patient and the family from going to the hospital during a pandemic, and have suggested clear guidelines for patient management in each subgroup of IEMs to be prepared in such circumstances (4).

Since ERT is given with frequent intervals (weekly or bi-weekly), the obligation to attend health-care facilities regularly, is not only time consuming, but also is unmanageable during times of overloading to the healthsystems. Home therapy should be encouraged in patients with stable conditions as LSDs, since it is an efficient and comfortable way to sustain treatment during pandemics. Another solution may be separation of specific centers for the care of patients with rare and chronic illnesses to be infusion centers, or sparing a safe are within centers for infusion. Since a shortage of health-care staff may also be present during an emergency state, developing protocols for the management of LSDs during pandemics is also necessary. Also, to ensure safe transport of patients to health centers may be helpful.

Several studies have shown that the COVID-19 pandemic has led to panic and anxiety in patients with chronic illnesses (17). Fiumara et al (18) have specifically analyzed the attitude of 15 patients with LSD towards the pandemic and the impact of the outbreak on their treatment. Parents of patients at pediatric ages were also involved in the study. Although no statistically significant difference was found compared to controls, all the evaluated persons revealed increase of anxiety related with COVID-19. The authors concluded that, the reactions of patients with LSD were qualitatively different than the general population, and they have stressed the fragility and isolation of such patients.

Similar to the studies in the literature, we have also determined depressive symptoms in the majority of our study group consisting of LSD patients and their parents. What is more interesting was that, a considerable number of parents with anxiety and depression, had interrupted their child's treatment, with the fear of getting infected. The same situation was observed among the adult patients, 
in which the patient with HADS scores indicative of both anxiety and depression, was the one who interrupted treatment. Our study indicates that, increased anxiety, along with depression, may aggravate the fear related with the pandemic, and may contribute to withdrawal of ERT. Thus, the identification of patients with anxiety and depression may be useful, since LSDs represent a vulnerable group of patients. Patient organizations may also be helpful, a part of care and support. The patients should be encouraged for the continuation of treatment.

\section{Study Limitations}

Our study has various limitations. First of all, a small number of patients were evaluated that may not reflect the whole population of LSDs that have been diagnosed and being treated in our country, and may not be generalized. Secondly, parental reports were evaluated for children under age 8 which may not directly reflect the child's own feelings.

\section{Conclusion}

Patients with LSDs represent a vulnerable group of the population due to the multisystemic effects of the disease. It is important to identify the problems these patients have been facing during the pandemic, also their attitudes regarding ERT in order to maintain the sustainability of their treatment. There is an urgent need to introduce homebased treatment options, where unavailable, to reduce the unfavourable health impacts due to disruptions of treatment. Also, individual guidelines for subtypes of LSDs should be created for the treatment strategies during the pandemic. Home-based therapy should be a choice for suitable patients since the effects of the pandemic seem to continue for an unpredictable period of time. The psychological health of these patients should also be identified, where needed, to provide optimal care to patients.

\section{Ethics}

Ethics Committee Approval: Ethics approval was obtained from the ethics committee of Yıldırım Beyazıt University, on September 2020 (number: 76).

Informed Consent: All participants provided informed consent.

Peer-review: Externally peer-reviewed.

\section{Authorship Contributions}

Surgical and Medical Practices: A.O., C.S.K, S.B.A., Y.Y., G.K.M., M.K., Concept: A.O., C.S.K, M.K., Design: A.O., C.S.K,
M.K., Data Collection or Processing: A.O., S.B.A, Y.Y., G.K.M., Analysis or Interpretation: A.O., S.B.A., Y.Y., M.K., Literature Search: A.O., S.B.A, Y.Y., G.K.M., Writing: A.O., C.S.K, S.B.A., Y.Y., G.K.M., M.K.

Conflict of Interest: No conflict of interest was declared by the authors.

Financial Disclosure: The authors declared that this study received no financial support.

\section{References}

1. WHO coronavirus (COVID-19) dashboard [Internet]. World Health Organ; 2020. [cited 2020 May 31]. Available from: https://who.sprinklr.com/.

2. Oni T, Micklesfield LK, Wadende P, et al. Implications of COVID19 control measures for diet and physical activity, and lessons for addressing other pandemics facing rapidly urbanizing countries. Glob Health Action 2020; 13:1810415.

3. https://www.nhsinform.scot/illnesses-and-conditions/ infections-and-poisoning/coronavirus-covid-19/coronaviruscovid-19-shielding.

4. Elmonem MA, Belanger-Quintana A, et al. The impact of COVID-19 pandemic on the diagnosis and management of inborn errors of metabolism: A global perspective. Mol Genet Metab 2020;25:S1096-7192:30195-5.

5. Solomon M, Muro S. Lysosomal enzyme replacement therapies: Historical development, clinical outcomes, and future perspectives. Adv Drug Deliv Rev 2017; 118:109-34.

6. Kanzaki M, Tsukimura T, Chiba $Y$, Sakuraba H, Togawa T. Surface plasmon resonance analysis of complex formation of therapeutic recombinant lysosomal enzymes with domain 9 of human cation-independent mannose 6-phosphate receptor. Mol Genet Metab Rep 2020;21;25:100639.

7. Chorpita BF, Yim L, Moffitt C, et al. Assessment of symptoms of DSM-IV anxiety and depression in children: a Revised Child Anxiety and Depression Scale. Behav Res Ther 2000; 38:835-55.

8. Gormez V, Kilincaslan A, Ebesutani C, et al. Psychometric Properties of the Parent Version of the Revised Child Anxiety and Depression Scale in a Clinical Sample of Turkish Children and Adolescents. Child Psychiatry Hum Dev 2017; 48:922-33.

9. Gormez V, Kılınçaslan A, Orengul AC, et al. Psychometric properties of the Turkish version of the Revised Child Anxiety and Depression Scale - Child Version in a clinical sample, Psychiatry and Clinical Psychopharmacology 2017; 27:84-92.

10. Aydemir O. Validity and Reliability of Turkish Version of Hospital Anxiety and Depression Scale. Turkish Journal of Psychiatry 1997; 8:280-87.

11. Zigmond AS, Snaith RP. The Hospital Anxiety and Depression Scale. Acta Psychiatrica Scandinavica 1983; 67:361-70.

12. Andrade-Campos MM, de Frutos LL, Ceblalla IJ, et al. Identification of risk features for complication in Gaucher's disease patients: a machine learning analysis of the Spanish registry of Gaucher disease. Orphanet I Rare Dis 2020; 15:256.

13. Miller $\mathrm{K}, \mathrm{McGrath} \mathrm{ME}, \mathrm{Hu} \mathrm{Z}$, et al. Coronavirus interactions with the cellular autophagy machinery. Autophagy. 2020:1-9. Online ahead of print. 
14. Mistry P, Balwani M, Barbouth D, et al. Gaucher disease and SARS-CoV-2 infection: Emerging management challenges. Mol Genet Metab. 2020; 130:164-69.

15. Politei J. Fabry disease during the COVID-19 pandemic. Why and how treatment should be continued. Mol Genet Metab 2020; 130:227-29.

16. Sechi A, Macor D, Valent S, et al. Impact of COVID-19 related healthcare crisis on treatments for patients with lysosomal storage disorders, the first Italian experience. Mol Genet Metab. 2020; 130:170-71.
17. Ademhan Tural D, Emiralioglu N, Tural Hesapcioglu S, et al. Psychiatric and general health effects of COVID-19 pandemic on children with chronic lung disease and parents' coping styles. Pediatr Pulmonol 2020;18. Epub ahead of print.

18. Fiumara A, Lanzafame G, Arena A, et al. COVID-19 Pandemic Outbreak and its Psychological Impact on Patients with Rare Lysosomal Diseases. J Clin Med 2020; 9:E2716. 